(2004) 000-000

HEAVY

ION

PHYSICS

\title{
From chemical freezeout to critical conditions in heavy ion collisions
}

\author{
Krzysztof Redlich ${ }^{1,2}$ \\ ${ }^{1}$ Institute of Theoretical Physics University of Wroclaw, \\ PL-50204 Wrocław, Poland \\ ${ }^{2}$ Fakultät für Physik, Universität Bielefeld, \\ Postfach 100 131, D-33501 Bielefeld, Germany
}

Received 1 January 2004

\begin{abstract}
We compare the statistical thermodynamics of hadron resonance gas with recent LGT results at finite chemical potential. We argue that for $T \leq$ $T_{c}$ the equation of state derived from Monte-Carlo simulations of two quarkflavor QCD at finite chemical potential is consistent with that of a hadron resonance gas when applying the same set of approximations as used in LGT calculations. We indicate the relation of chemical freezeout conditions obtained from a detailed analysis of particle production in heavy ion collisions with the critical conditions required for deconfinement. We argue that the position of a hadron-quark gluon boundary line in temperature chemical potential plane can be determined in terms of the resonance gas model by the condition of fixed energy density.
\end{abstract}

Keywords: Heavy ion collisions

PACS:

\section{Introduction}

A detailed analysis of particle production in heavy ion collisions has shown that in a broad energy range from SIS through AGS, SPS ${ }^{1}$ up to RHIC particle yields resemble that of chemical equilibrium population with respect to the partition function of hadron resonance gas (HRG) [1].

In Fig. (11) we show an example of the comparison of HRG-model with experimental data on different particle yields ratios measured in $\mathrm{Au}-\mathrm{Au}$ collisions at RHIC energies. The model results [ [3, 4] are shown as horizontal lines whereas

\footnotetext{
${ }^{1}$ The most recent thermal analysis of SPS data was presented in Ref. [ 2].
} 
experimental data are indicated as points. The apparent quite good agreement of the model predictions with the experiment indicates that production of particles in heavy ion collisions is of thermal origin [ [5]. A collision fireball at chemical freezeout is characterized by only two thermal parameters, the temperature $T$ and baryon chemical potential ${ }^{2} \mu_{B}$. The volume of the fireball can be possibly connected with the HBT source size [ [6, 7].

Figure (11) shows the compilation of the chemical freeze-out parameters that are required to reproduce the measured particle yields in central $\mathrm{Au}-\mathrm{Au}$ or $\mathrm{Pb}-$ $\mathrm{Pb}$ collisions at SIS, AGS, SPS and RHIC energy. The GSI/SIS results have the lowest freeze-out temperature and the highest baryon chemical potential. As the beam energy increases a clear shift towards higher $T$ and lower $\mu_{B}$ occurs. There is a common feature to all these points, namely that the average energy $\langle E\rangle$ per average number of hadrons $\langle N\rangle$ is approximately $1 \mathrm{GeV}$. A chemical freeze-out in $\mathrm{A}-\mathrm{A}$ collisions is thus reached [8] when the energy per particle $\langle E\rangle /\langle N\rangle$ drops below $1 \mathrm{GeV}$ at all collision energies. The physical origin of the above freezeout condition requires dynamical justification. Recently, this point has been investigated in central $\mathrm{Pb}-\mathrm{Pb}$ collisions at the SPS in terms of the Ultra-relativistic Quantum Molecular Dynamics model (UrQMD) [ 9 10]. A detailed study has shown that there is a clear correlation between the chemical break-up in terms of inelastic scattering rates and the rapid decrease in energy per particle. If $\langle E\rangle /\langle N\rangle$ approaches the value of $1 \mathrm{GeV}$ the inelastic scattering rates drop substantially and further evolution is due to elastic and pseudo-elastic collisions that preserved the chemical composition of the collision fireball. Following these UrQMD results one could consider the phenomenological chemical freeze-out of $\langle E\rangle /\langle N\rangle \simeq 1 \mathrm{GeV}$ as the condition of inelasticity in heavy ion collisions.

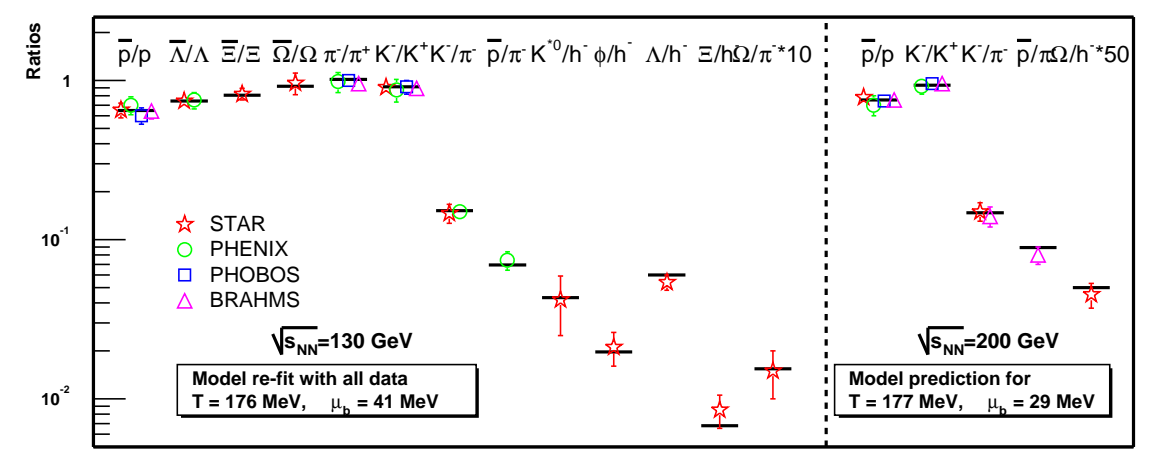

Fig. 1. Comparison of the experimental data on different particle multiplicity ratios obtained at RHIC at $\sqrt{s}_{N N}=130$ and $200 \mathrm{GeV}$ with thermal model calculations. The thermal model analysis is from Refs. [3, 4].

\footnotetext{
${ }^{2}$ The quark chemical potential $\mu_{q}=\frac{1}{3} \mu_{B}$
} 


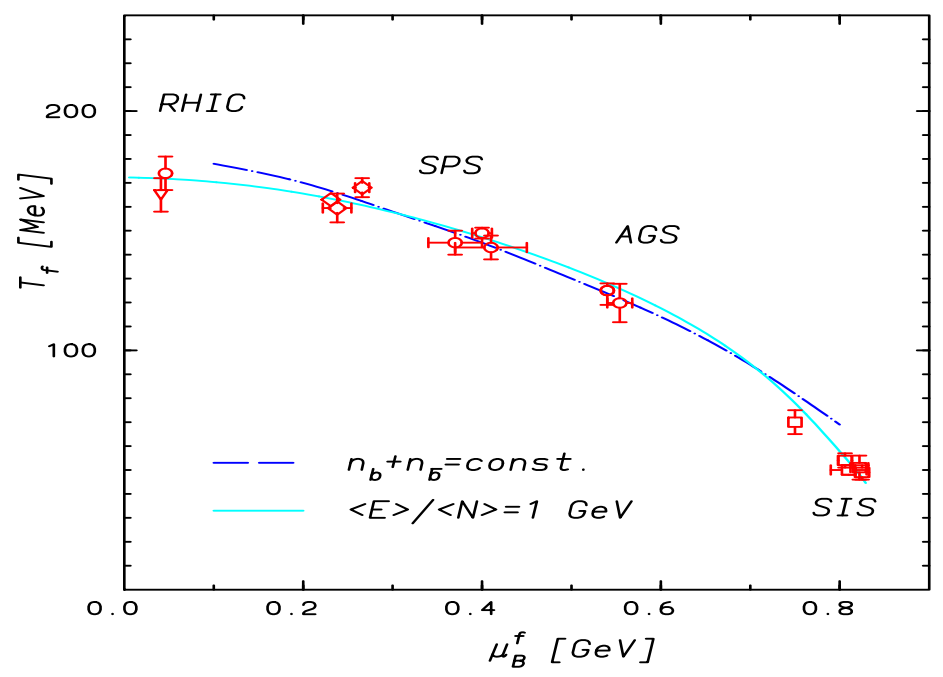

Fig. 2. Chemical freezeout in heavy ion collisions. The full line represents the condition of fixed energy/particle $\simeq 1.0 \mathrm{GeV}$ from Ref. [ [8]. The broken line describes the chemical freeze-out conditions of fixed total density of baryons plus antibaryons, $n_{b}+n_{\bar{b}}=0.12 / \mathrm{fm}^{3}$ from Ref. [11]. The points are the average values of $T_{f}$ and $\mu_{B}^{f}$ obtained from the analysis of particle yields in heavy ion collisions at the SIS, AGS, SPS and RHIC energy [1].

Chemical freeze-out in heavy ion collisions was recently proposed to be determined [11] by the condition of fixed density of the total number of baryons plus antibaryons $F \equiv\left(n_{B}+n_{\bar{B}}\right) \simeq 0.12 / \mathrm{fm}^{3}$. It is seen in Fig. (2) that, within statistical uncertainties on the freeze-out parameters the above condition provides a good description of experimental data from the top AGS up to RHIC energy. However, at the low SIS energy it overestimates the freeze-out temperature for a given chemical potential. Consequently, e.g. the yield of pion/proton or pion/participant and strange/non-strange particle ratios obtained at SIS turn out to be larger than the experimental values. Taking the value of $F \sim 0.05 / \mathrm{fm}^{3}$ at SIS energy and using the freezeout condition of $F_{S I S}=$ fixed leads eg. at RHIC to the freezeout temperature of $\sim 150 \mathrm{MeV}$, the value which is lower than obtained from the particle yield ratios as seen in Fig. (11). At $\mu_{B}=0$ the $\langle E\rangle /\langle N\rangle$ condition converge to $T_{f} \simeq 170$ $\mathrm{MeV}$ which is well consistent with the critical temperature $T=167(13)$ [12] and $T=173(8)$ [ [13] expected in $(2+1)$ and 2-flavour QCD respectively.

At SPS and RHIC the freeze-out parameters, the temperature $T_{f}$ and the energy density $\epsilon_{f}$, predicted by the presently used thermal model of hadron resonance gas, agree well with the values of the corresponding parameters required for deconfinement in LGT calculations [1, 14, 15]. The above quantitative agreement 
of freeze-out and critical parameters suggests that at SPS and RHIC the chemical freeze-out appears in the near vicinity of the phase boundary [18]. If this is indeed the case then the phenomenological statistical operator of hadron resonance gas $Z_{H G}$ should also provide, consistent with LGT, description of QCD thermodynamics in confined, hadronic phase of QCD [15, 16, 17, 19]. In the following we argue that the basic qualitative properties of $Z_{H G}$ resulting from its dependence on $T$ and baryon chemical potential $\mu_{B}$ are indeed present in the recent lattice results of two flavor QCD at the finite chemical potential. We also show that imposing a fixed energy density condition for deconfinement the hadron resonance gas partition function $Z_{H G}$ can also describe [15] the quark mass and number of flavor dependence of the lattice critical temperature at $\mu_{B}=0$ and the position of the phase boundary in the $\left(T, \mu_{B}\right)$-plane at small $\mu_{B}[20$.
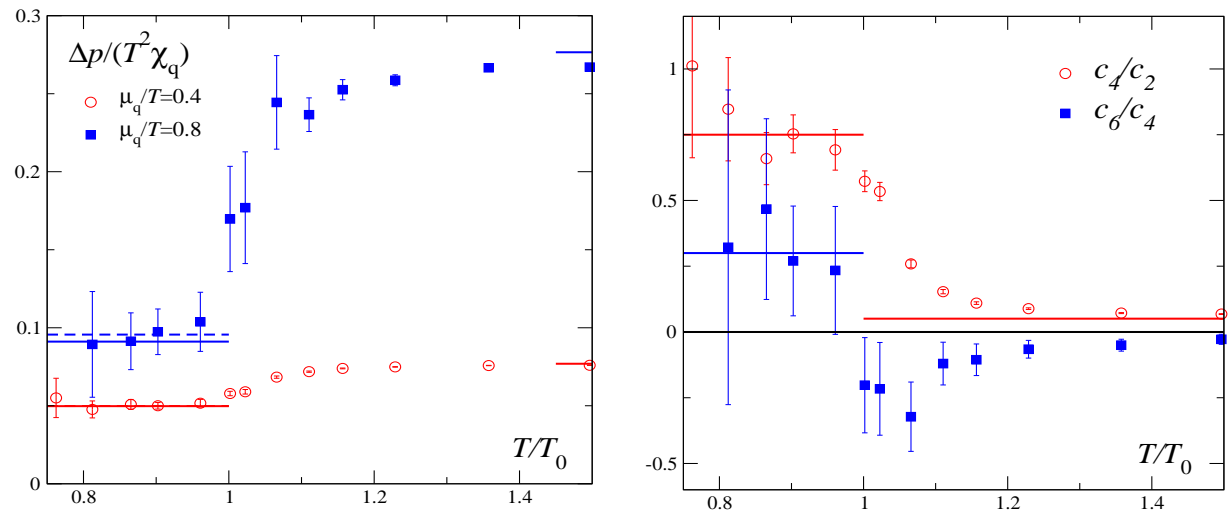

Fig. 3. Left-hand figure: temperature dependence of $\Delta P /\left(\chi_{q} T^{2}\right)$ ratio for two different values of $\mu_{q} / T$. The 2-flavor lattice results are from [ 23] 24]. The lines are the hadron resonance gas model values with (broken-line) and without (full-line) the Taylor expansion of $\left.\cosh \left(\mu_{B} / T\right)\right)$ [ [15]. The right-hand side shows the temperature dependence of the ratios of the second, fourth and sixth order coefficients in the Taylor expansion of thermodynamic pressure. The points are lattice results from [23, 24]. The lines at $T<T_{0}$ are the values obtained from the expansion of $\left.\cosh \left(\mu_{B} / T\right)\right)$.

\section{QCD thermodynamics of hadronic phase}

The phenomenological partition function used in the description of particle productions in heavy ion collisions was, following Hagedorn, constructed as a noninteracting hadronic gas which is composed of all hadrons and resonances. In this approach the interactions between hadrons are included to the extent that the thermodynamics of an interacting system of elementary hadrons is effectively approximated by that of mixture of ideal gases of stable particles and resonances [ 21, 22]. 
In the Boltzmann approximation, suitable for the moderate values of $\mu_{B}<m_{N}$ and $T \geq 50 \mathrm{MeV}$, there is a factorization of $T$ and $\mu_{B} / T$ dependence in relevant observables characterizing a baryonic sector of the system. The basic quantity is the pressure $\Delta P=P\left(T, \mu_{B}\right)-P\left(T, \mu_{B}=0\right)$

$$
\frac{\Delta P}{T^{4}} \simeq F(T)\left(\cosh \left(\frac{\mu_{B}}{T}-1\right) \quad \text { with } \quad F(T) \simeq \int d m \rho(m)\left(\frac{m}{T}\right)^{2} K_{2}\left(\frac{m}{T}\right)\right.
$$

from which the baryon density $n_{B}$ and the baryon number susceptibility $\Delta \chi_{B}$ are obtained as the first and second order derivatives with respect to $\mu_{B}$, respectively. In the phenomenological application of the above model the mass spectrum $\rho(m)$ in Eq. (1) is approximated as the sum of delta functions. The baryonic pressure gets the contributions from all known baryons and resonances.

The net baryonic pressure $\Delta P\left(T, \mu_{q}\right)$ has been recently obtained on the lattice in two flavor QCD as the Taylor series in $\mu_{q} / T$

$$
\frac{\Delta p\left(T, \mu_{q}\right)}{T^{4}} \simeq \sum_{n=1}^{n=3} c_{2 n}(T)\left(\frac{\mu_{q}}{T}\right)^{2 n} .
$$

up to $O\left(\mu_{q}^{6}\right)$ order [23, 24].

The basic observables characterizing baryonic contribution to the QCD thermodynamics, the net quark density $n_{q}$ and susceptibility $\chi_{q}$ are obtained from Eq.(2) as

$$
\begin{aligned}
& \frac{n_{q}}{T^{3}}=\frac{\partial \Delta P\left(T, \mu_{q}\right) / T^{4}}{\partial \mu_{q} / T} \simeq 2 c_{2}(T)\left(\frac{\mu_{q}}{T}\right)+4 c_{4}(T)\left(\frac{\mu_{q}}{T}\right)^{3}+6 c_{6}(T)\left(\frac{\mu_{q}}{T}\right)^{5} \\
& \frac{\chi_{q}}{T^{2}}=\frac{\partial^{2} \Delta p\left(T, \mu_{q}\right) / T^{4}}{\partial\left(\mu_{q} / T\right)^{2}} \simeq 2 c_{2}(T)+12 c_{4}(T)\left(\frac{\mu_{q}}{T}\right)^{2}+30 c_{6}(T)\left(\frac{\mu_{q}}{T}\right)^{4} .
\end{aligned}
$$

The coefficients $c_{i}(T)$ in the Taylor expansion (213) were calculated through the Monte-Carlo simulations of 2-flavor QCD [23, 24].

The lattice results (2 3) restricted to the confined phase of QCD can be directly compared with the predictions of the hadron resonance gas model (11). This, in general, required a numerical analysis. However, there are particular features of the resonance gas partition function that could be directly verified by the LGT results.

First, as seen from Eq. (11) there is a factorization of $T$ and $\left(\mu_{q} / T\right)$ dependence in the thermodynamic pressure and associated baryonic observables $n_{q}$ and $\chi_{q}$. Thus, in the resonance gas model any ratios of these observables, calculated at fixed quark chemical potential $\left(\mu_{q} / T\right)=$ fixed, should be independent of temperature [ 15. 19]. In Fig. (3-left) we show as an example the ratio of $\Delta P / \Delta\left(\chi_{B} T^{2}\right)$ for two different values of $\mu_{q} / T$ as the function of $T$. It is clear from Fig. (3-left) that the factorization predicted by HRG partition function $Z_{H G}$ is also seen in LGT results for $T<T_{c}$.

Second, the $\left(\mu_{q} / T\right)$-dependence in the HRG-model is described by the (cosh)function. Thus, performing the Taylor expansion of the pressure (10) in $\left(\mu_{q} / T\right)$ 


$$
\frac{\Delta P\left(T, \mu_{q}\right)}{T^{4}} \simeq F(T)\left(\frac{9}{2}\left(\frac{\mu_{q}}{T}\right)^{2}+\frac{81}{4 !}\left(\frac{\mu_{q}}{T}\right)^{4}+\frac{3^{6}}{6 !}\left(\frac{\mu_{q}}{T}\right)^{6}\right)
$$

one gets the predictions on the values of the coefficients $c_{i}$ in (3) from the HRGmodel

$$
c_{2}(T)=\frac{9}{2} F(T), c_{4}=\frac{81}{4 !} F(T), c_{6}=\frac{3^{6}}{6 !} F(T) .
$$

where for a discrete hadronic mass spectrum

$$
F(T)=\sum_{i} \frac{d_{i}}{\pi^{2}}\left(\frac{m_{i}}{T}\right)^{2} K_{2}\left(\frac{m_{i}}{T}\right) .
$$

with the sum taken over all baryons and baryonic resonances of mass $m_{i}$ and the spin-isospin degeneracy factor $d_{i}$.

In Fig. (3-right) we show the ratios $c_{4} / c_{2}$ and $c_{6} / c_{4}$ obtained on the lattice in 2-flavour QCD and the corresponding results of the HRG-model from Eq. (5): $c_{4} / c_{2}=3 / 4$ and $c_{6} / c_{4}=0.3$. The temperature dependence of the Taylor coefficients in Eq. (414) and (5) is controlled by a common function for all $c_{i}$. Thus, in HRGmodel, the ratios of different $c_{i}$ are independent of $T$. The lattice results for $T<T_{c}$ are, within statistical errors, consistent with this prediction. The values of $c_{6} / c_{4}$ and $c_{4} / c_{2}$ ratios obtained recently on the lattice [23, 24] are also seen in Fig. (3right) to coincide with HRG-model. At temperature $T \simeq T_{c}$, however, the lattice results deviate from the resonance gas values. This is to be expected as at the critical temperature, deconfinement releases the color degrees of freedom ${ }^{3}$ which are obviously not present in the statistical operator of the hadron resonance gas. The partition function (11) is not sensitive to the phase transition thus, could be at most applicable below deconfinement.

The pressure calculated on the lattice (see Fig. (4) ) increases abruptly when approaching deconfinement transition from the hadronic side. If the phenomenological statistical operator $Z_{H G}$ is of physical significance then this increase could be due to the resonance formation. To check the importance of resonances near deconfinement one would need to reproduce lattice results on the $T$-dependence of $\Delta P$ at fixed $\mu_{q} / T$. However, to quantify this dependence one needs to implement the same set of approximations in Eq.(1) as those being used on the lattice. The lattice results were obtained in 2-flavor QCD, thus there is no contribution of strange baryons in Eq.(1). In addition due to the Taylor expansion of $\Delta P$ in the lattice calculations one also needs to apply a similar approximation in Eq.(1). 25].

${ }^{3}$ These color degrees of freedom could be also possibly described as resonance bound states [ 


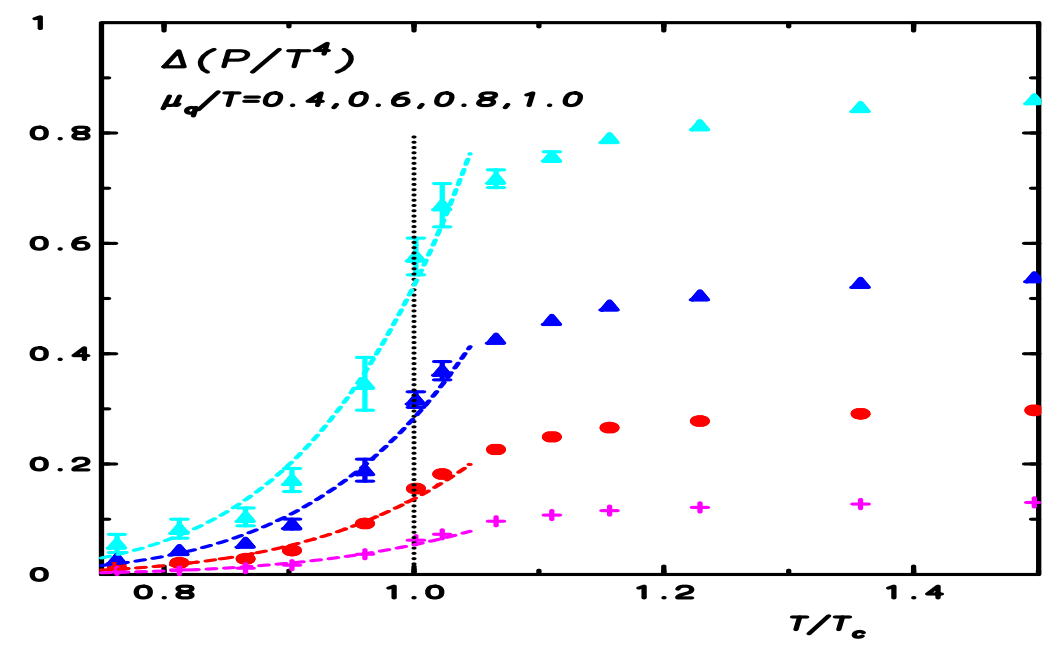

Fig. 4. Temperature dependence of baryonic pressure for different values of $\mu_{q} / T$. The lattice results are from [23]. The lines are the statistical model results [15].

The current lattice results in Fig. (4) were obtained with a large quark mass corresponding to $m_{\pi} \simeq 770 \mathrm{MeV}$. This large quark mass distorts the baryon mass spectrum in Eq. (11). The pion mass dependence of baryon masses can be obtained from lattice calculations at zero temperature [15, 26]. We use the following ansatz for the parametrization of baryon mass dependence on the pion mass,

$$
\frac{m^{*}\left(m_{\pi}\right)}{m} \simeq 1+A \frac{m_{\pi}}{m^{2}},
$$

with $A=0.9 \pm 0.1, m^{*}$ being a distorted hadron mass at fixed $m_{\pi}$ and $m$ is its corresponding physical value.

In Fig. (4) the lattice results are compared with Eq. (11) calculated with modified baryon mass spectrum following Eq. (7). The $T$ dependence of QCD thermodynamics obtained on the lattice at different values of $\mu_{q} / T$ is seen in Fig. (4) to be well consistent with the predictions of a HRG-model. A similar agreement was also found for other relevant thermodynamic observables in (2+1), 3-flavour [ 15] and 4-flavour QCD [ 16]. The above agreements indicate that the phenomenological partition function of hadron resonance gas should be a good approximation of the QCD statistical operator in the hadronic phase.

\section{Critical conditions for deconfinement}

The recent lattice results on QCD thermodynamics show that deconfinement occurs at similar values of the energy density in QCD with light quarks as well as in the pure gauge theory, although the transition temperature shifts by about $40 \%$ and $\epsilon / T_{c}^{4}$ differs by an order of magnitude [13]. Thus, it has been suggested that 

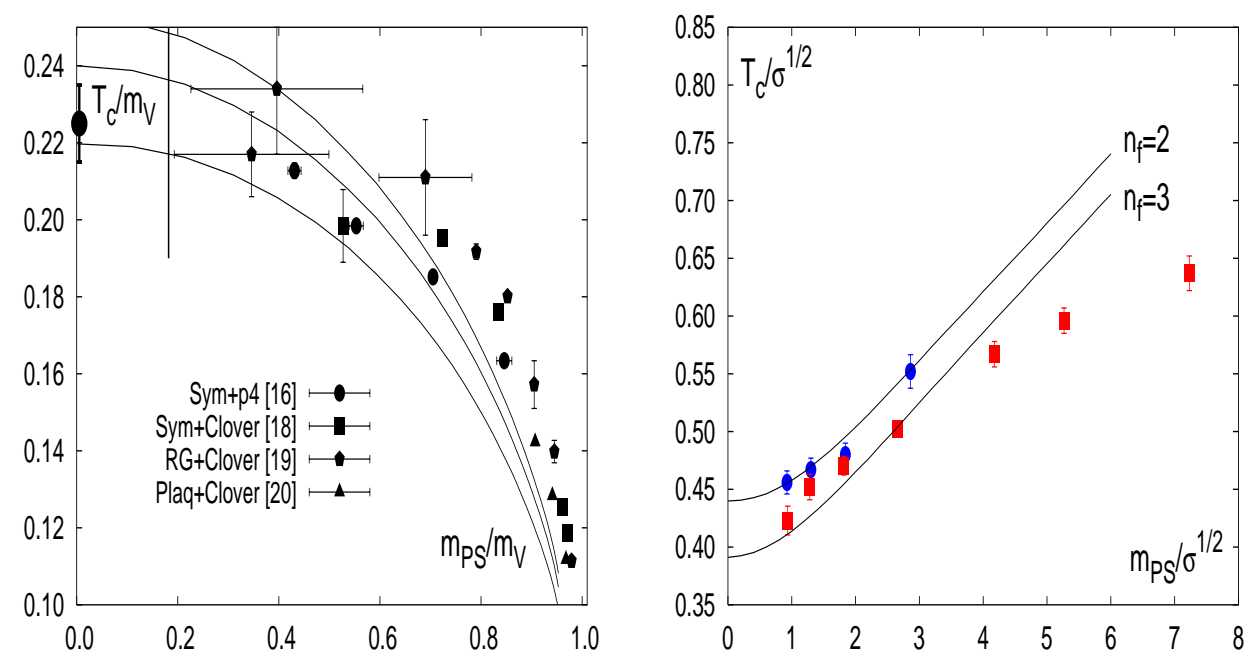

Fig. 5. The transition temperature vs. pion mass obtained in lattice calculations and lines of constant energy density calculated in a resonance gas model [15]. The left hand figure shows a comparison of constant energy density lines at 1.2 (upper), 0.8 (middle) and 0.4 (lower) $\mathrm{GeV} / \mathrm{fm}^{3}$ with lattice results for 2-flavor QCD obtained with improved staggered [13] as well as improved Wilson [ 27, 28, 29] fermion formulations. $T_{c}$ as well as $m_{P S}$ are expressed in terms of the corresponding vector meson mass. The right hand figure shows results for 2 and 3 flavor QCD compared to lines of constant energy density of $0.6 \mathrm{GeV} / \mathrm{fm}^{3}$. Here $T_{c}$ and $m_{P S}$ are expressed in units of $\sqrt{\sigma}$. For a detailed description see Ref. [15].

for arbitrary quark masses the transition in QCD appears at the roughly constant energy density. Such an assumption can be verified by the HRG-model formulated for an arbitrary value of the quark mass [15]. In Fig. (5) we show the lines of the constant energy density calculated in the resonance gas model and compare these to the transition temperatures obtained in lattice calculations. As can be seen the agreement is quite good up to masses, $m_{\pi} \simeq 3 \sqrt{\sigma}$ or $m_{\pi} \simeq 1.2 \mathrm{GeV}$. At larger values of the quark mass the observed deviations are due to the fact that thermodynamics is dominated by glueballs which are not present in the hadronic mass spectrum [15].

The critical conditions of fixed energy density could be also verified at the finite chemical potential. ${ }^{4}$ Fig. (6) shows recent lattice results on the position of the phase boundary line in 2-flavor QCD obtained within the Taylor approximation and with the pion mass $m_{\pi} \simeq 770 \mathrm{MeV}$. The line of fixed energy density $\epsilon \simeq 0.6 \mathrm{GeV} / \mathrm{fm}^{3}$ with $\epsilon$ calculated in the HRG-model with the modified mass spectrum is seen to

\footnotetext{
${ }^{4}$ The critical condition of fixed energy density can be related with the percolation condition for deconfinement [30].
} 


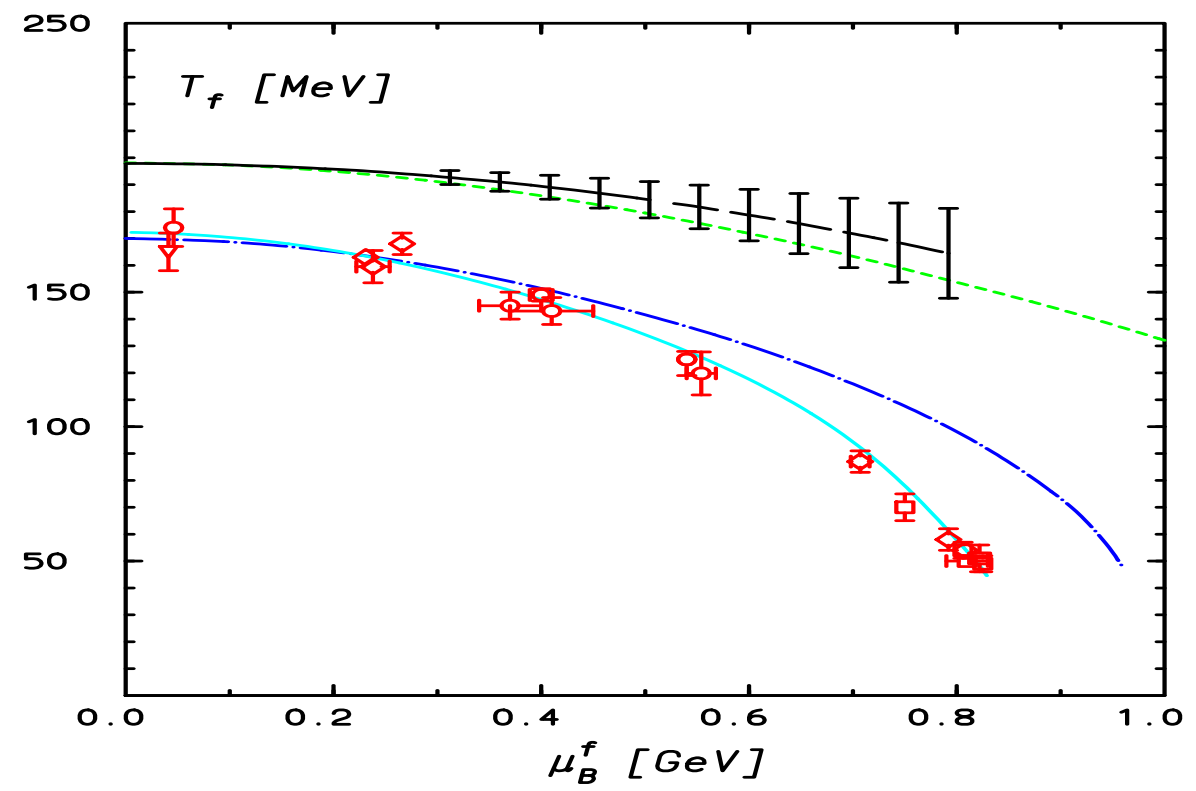

Fig. 6. Lattice results [23] on the phase boundary curve (line with errors) together with phenomenological freeze-out values of $T$ and $\mu_{B}$ (points) obtained from the analysis of particle production in heavy ion collisions [1]. Short-dashed and dasheddotted lines are the statistical model results obtained under the condition of fixed $\epsilon \simeq 0.6 \mathrm{GeV} / \mathrm{fm}^{3}$ with $m_{\pi} \simeq 0.77 \mathrm{GeV}$ and $m_{\pi} \simeq 0.14 \mathrm{GeV}$ respectively. Also shown (full-line) is the phenomenological freeze-out curve of fixed energy/particle $\simeq$ $1 \mathrm{GeV}$ from [8].

coincide with lattice results. Decreasing the pion mass to its physical value and including a complete set of resonances expected in $(2+1)$-flavor QCD results in the shift of the position of the phase boundary line towards a phenomenological freezeout condition of the fixed energy/particle $\simeq 1 \mathrm{GeV}$. The splitting of freeze-out and phase boundary line appears when the ratio of meson/baryon multiplicities reaches the unity.

The dashed-dotted line in Fig. (6) could be a physical boundary line separating the hadronic from quark-gluon plasma phase. However, in the baryon dominating medium one expects a very efficient meson baryon scatterings [31]. Consequently, the particle dispersion relations and spectral functions can be modified in a medium. Thus, it is not clear if in this case the partition function of hadron resonance gas with free particle dispersion relations is still an adequate approximation of the QCD thermodynamics just before deconfinement. In view of the above, the position of the critical curve shown in Fig. (6) could be shifted in the parameter range where $\mu_{B}>0.5 \mathrm{GeV}$, that is where baryons start to be dominating degrees of freedom in a thermal fireball. 


\section{Conclusions}

We have shown that the statistical operator of hadron resonance gas used to describe particle yields in heavy ion collisions provides a satisfactory description of the recent lattice results on QCD thermodynamics at the finite chemical potential. In particular, the basic property of this operator like e.g. a factorization of temperature and chemical potential dependence is obviously confirmed by the lattice results. The ratios of the coefficients in the Taylor expansion of a thermodynamic pressure obtained in LGT are as well described by the expansion of cosh-function predicted by the hadron resonance gas. These results should be independent from the particular choice of the quark mass in lattice calculations and are also to a large extent free from lattice artifacts. The phenomenological partition function, with $m_{\pi}$-dependent hadronic mass spectrum, was also shown to describe quantitatively the temperature and chemical potential dependence of the basic thermodynamical observables obtained in LGT for $T<T_{c}$. This indicates that the hadron resonance gas partition function is a good approximation of QCD thermodynamics in the hadronic phase. Applying the above partition function together with the condition

of a fixed energy density we have discussed a possible position of the QCD critical curve in the temperature-chemical potential plane.

\section{Acknowledgments}

Stimulating discussions with P. Braun-Munzinger, S. Ejiri, F. Karsch, H. Satz, J. Stachel and A. Tawfik are kindly acknowledged. This work was partly supported by the KBN under grant 2P03 (06925) and by the DFG under grant GRK 881.

\section{References}

1. For review see: P. Braun-Munzinger, K. Redlich and J. Stachel, in Quark Gluon Plasma 3 (Edts. R. Hwa and X.-N. Wang); nucl-th/0304013

A. Andronic and P. Braun-Munzinger, hep-ph/0402291.

2. J. Manninen, F. Becattini, A. Keranen, M. Gazdzicki and R. Stock, nucl-th/0405015

3. P. Braun-Munzinger, D. Magestro, K. Redlich, and J. Stachel, Phys. Lett. B518 (2001) 41 and references therein.

4. D. Magestro, J. Phys. G28 (2002) 1745.

5. See also, M. Gyulassy and L. McLerran, nucl-th/0405013

6. G. Agakichiev et al., CERES/NA45 Collaboration, Phys. Rev. Lett. 92 (2004) 032301.

7. H. Satz, hep-ph/0405051

8. J. Cleymans, and K. Redlich, Phys. Rev. Lett. 81 (1998) 5284.

9. M. Bleicher and J. Aichelin, Phys. Let. B530 (2002) 81. 
10. see also: L. Bravina, et al., Nucl. Phys. A698 (2002) 383; Phys. Rev. C66 (2002) 014906.

11. P. Braun-Munzinger and J. Stachel, J. Phys. G28 1971 (2002).

12. C. Bernard et al., MILC Collaboration, hep-lat/0405029

13. F. Karsch, E. Laermann, A. Peikert, Nucl. Phys. B605 (2001) 579; F. Karsch, E. Laermann, A. Peikert, Phys. Lett. B478 (2000) 447; F. Karsch, Nucl. Phys. A698 (2002) 199c.

14. J. Stachel, Nucl. Phys. A654 (1999) 119c.

15. F. Karsch, K. Redlich and A. Tawfik, Eur. Phys. J. C29 (2003) 549;

F. Karsch, K. Redlich and A. Tawfik, Phys. Lett. B571 (2003) 67.

16. M. D'Elia and Maria-Paola Lombardo, GEF-TH-2004-07; hep-lat/0406012

17. M. D'Elia, Maria-Paola Lombardo, Phys. Rev. D67 (2003) 014505; Maria-Paola Lombardo, hep-lat/0210040

18. P. Braun-Munzinger, and J. Stachel, Nucl. Phys. A606 (1996) 320; Nucl. Phys. A683 (1998) 3.

19. S. Ejiri, et al., to appear.

20. F. Karsch, K. Redlich and A. Tawfik, nucl-th/0404009 A. Tawfik, et al., to appear.

21. R. Hagedorn, Nuovo Cimento 35 (1965) 395; R. Hagedorn, Thermodynamics of strong interactions, CERN Report 71-12 (1971).

22. R. Vanugopalan and M. Prakash, Nucl. Phys. A546 718 (1992); V. Koch, Nucl. Phys. A715 108 (2003).

23. C. R. Allton, S. Ejiri, S. J. Hands, O. Kaczmarek, F. Karsch, E. Laermann and C. Schmidt, Phys. Rev. D68 (2003) 014507; see also, S. Ejiri, poster session at Quark Matter 04; S. Ejiri, hep-lat/0401012.

24. C. Allton, et al., to appear.

25. E. Shuryak, hep-ph/0405066 and references therein.

26. M. Göckeler, R. Horsley, D. Pleiter, P. Rakow and G. Schierholz Phys. Lett. B532 (2002) 63; C. M. Maynard, Nucl. Phys. Proc. Suppl. 10989.

27. C. Bernard, et al., Phys. Rev. D56 (1997) 5584, and references therein.

28. A. Ali Khan et al. (CP-PACS), Phys. Rev. D63 (2001) 034502.

29. R.G. Edwards and U.M. Heller, Phys. Lett. B462 (1999) 132.

30. V. Magas and H. Satz, Eur. Phys. J. C32 (2003) 115.

31. W. Weinhold, B. Friman and W. Nörenberg, Phys. Lett. B433, (1998) 236; M. Post, S. Leupold and U. Mosel, nucl-th/0309085 M.F.M. Lutz, G. Wolf and B. Friman, Acta Phys. Hung. New Ser. Heavy Ion Phys. 17 (2003) 313; Prog. Theor. Phys. Suppl. 149 (2003) 152; G.E. Brown, Guo-Qiang Li, R. Rapp, M. Rho and J. Wambach, Acta Phys. Polon. B29 (1998) 2309; G. E. Brown and M. Rho, Phys. Rept. 269333 (1996). 